\title{
In Vivo Magnetic Resonance Techniques and Drug Discovery
}

\author{
Nicolau Beckmann \\ Novartis Institutes for BioMedical Research, Discovery Technologies, \\ Lichtstr. 35, WSJ-386.2.09, CH-4002 Basel, Switzerland
}

Received on 5 October, 2005; accepted on 5 November, 2005

\begin{abstract}
The long and resource intensive process of drug discovery and development is confronted with the basic challenge of providing effective and safe therapies at reasonably low costs. The better the mechanism of a disease is known, the higher the probability to find an appropriate therapy. Also, the better and earlier a disease can be diagnosed and characterized, the higher the chance to be able to interfere in this process with a chemical entity. This reasoning sets the framework for the use of imaging in drug discovery. We discuss the relevance of magnetic resonance imaging and spectroscopy to derive anatomical, functional, metabolic and target-related information in the context of pharmacological research in vivo.
\end{abstract}

Keywords: Magnetic resonance; Imaging; Drug discovery

\section{INTRODUCTION}

The process to discover and to bring a drug to market is complex, costly and long, requiring in average 12 years. It begins with the identification and validation of a potential drug target. High affinity binders are searched for by using high-throughput screening (HTS). Compounds that have passed some initial selectivity filters are then further evaluated. Despite recent significant investments in biology related areas such as genomics and proteomics, and in technology platforms designed to increase the number of compounds assayed, the development time and attrition rate in clinical trials remained unchanged.

It is expected that improvements in the chemistry should reduce the attrition rate, thus increasing the success rate and, perhaps, reducing the development costs at the same time [1]. A much better understanding of this structure/performance relationship is needed to develop predictive algorithms that will increase the survival rate of compounds in clinical trials. Important features to be considered are absorption, distribution, metabolism, excretion and toxicology (ADMET) - all associated with the molecular structure.

\section{IMAGING IN DRUG RESEARCH}

Another important attempt in shortening the drug discovery and development process is related to improving the characterization of compounds and their effects in early and not yet so costly phases, and to transferring this knowledge into the clinical phase of testing. Intimately linked to this reasoning stand the knowledge about a given disease or disease model, and their early diagnosis and characterization. For sure, the better the etiology of a disease is known, the higher the chance to find an appropriate therapy. Also, the better a disease can be diagnosed and characterized, the higher the chance to be able to interfere in this process with a chemical entity. Advances in the understanding of disease progression at the cellular and molecular levels, which spur the development of drugs that are highly specific for their molecular target, along with progress in bioanalytical assay technologies constitute an attractive basis for choosing, describing and evaluating new biomarkers.

According to the Food and Drug Administration (FDA), a biomarker is a characteristic that is objectively measured and evaluated as an indicator of normal biological processes, pathogenic processes, or pharmacologic responses to a therapeutic intervention [2]. In our context, a biomarker can be considered a bioanalytical readout with diagnostic and prognostic quality that can be used for the identification of a pathology, monitoring of its progression, and for the evaluation of therapeutic interventions. Criteria for validating biomarkers include considerations of mechanistic plausibility, available methods and technologies, and preclinical and clinical feasibility. This link between preclinical and clinical studies demands for non-invasive bioanalytical technologies such as imaging. Validated biomarkers may be used for identifying patient populations, as well as for providing evidence of drug efficacy and potential toxicity (see section 4). The development of imaging strategies that meet the requirements for use in a clinical setting will facilitate the translation from animal models to human subjects, by minimizing changes in experimental paradigms while the model organism is changed [3-6]. What sets imaging biomarkers apart from e.g. analyses from blood serum and urine, used for decades in medicine and in drug development, or recently proposed proteomics biomarkers, is the fact that imaging readouts tend to be much more closely related to the disease phenotype, thus facilitating direct associations between therapy and effect.

The non-invasive character of imaging enables one to address several questions concerning drug discovery and development. For sure, no single imaging modality can answer all possible questions in this complicated domain. An important initial step is to find out which potential a given imaging modality has in addressing issues regarding a certain disease area. A complete review of imaging within pharmaceutical research is beyond the scope of this paper. Table 1 provides an overview of current imaging modalities of interest for drug research. In the next sections we are focusing our attention on magnetic resonance (MR) techniques. 


\section{BENEFITS AND LIMITATIONS OF IN VIVO MR TECHNIQUES}

The principal assets of magnetic resonance imaging (MRI) are non-invasiveness, high spatial resolution, of the order of $100 \mu \mathrm{m}$ for rodent studies, and excellent soft tissue contrasting capabilities. The MR signal is governed by a number of parameters, e.g., proton density, relaxation times $\left(T_{1}, T_{2}, T_{2} *\right)$, proton exchange rates, water diffusion, macroscopic motion (blood flow), which depend on the biophysical properties of the tissue. This wealth of information renders MRI a valuable tool for diagnosis, tissue staging and in vivo morphometry, for obtaining physiological and functional readouts, and for deriving metabolic and, to some extent, target-specific tissue characteristics (see section 6 below) in a non-invasive manner.

A major limitation of MR is its low sensitivity, which significantly determines the possible roles of the technique in pharmaceutical research. A simple calculation illustrates the fact that MR is, generally speaking, not suited for directly assessing the distribution of drugs in the organism [7]. A compound of molecular mass 500 administered at a dose of $1 \mathrm{mg} / \mathrm{kg}$ and evenly distributed throughout the body will result in an approximately $2 \mu \mathrm{M}$ tissue concentration (neglecting drug elimination). Nuclear medicine techniques such as single photon emission computed tomography (SPECT) or positron emission tomography (PET) and, more recently, near-infrared reflectance fluorescence optical imaging provide the required sensitivity to detect compounds in micromolar concentrations. Yet, these methods are hampered by a relatively low spatial resolution (in best cases, of about $1 \mathrm{~mm}$ ), which although acceptable in clinical applications turns out to be limiting when studying small animals, and a lack of chemical specificity, being therefore unable to distinguish whether the emitting reporter group is attached to the parent drug molecule or a metabolite. In vivo MR methodologies on the other hand require tissue concentrations in the millimolar range. The signals of a few endogenous metabolites can be observed and until now in exceptional cases only the fate of a drug in the target organ could be monitored using magnetic resonance spectroscopy (MRS). For instance, ${ }^{19} \mathrm{~F}$ MRS has been successfully applied to assess the pharmacokinetics of fluorinated drugs [8-11], and ${ }^{13} \mathrm{C}$ MRS to detect the distribution of ${ }^{13} \mathrm{C}$ labeled compounds in tumors $[12,13]$. These examples have been essentially limited to cancer therapeutics administered at high concentrations [14]. How many of such compounds can be administered at doses sufficiently high to be detectable in vivo by MR is unknown. Moreover, spatial resolution in these studies is poor, significantly inferior to nuclear approaches. Therefore, in general terms, the role of MRI/S in pharmacological research is to study the effects of a drug on tissue morphology, physiology and biochemistry rather than to study the fate of the drug itself in the organism; in other words MR methods yield pharmacodynamic and not a pharmacokinetic readouts [15-19].

Another limitation of MR methods is quantification. While absolute values of structural parameters (e.g. volumes of organs) are readily attainable, assessments of absolute physiological parameters from MRI data or absolute concentrations of metabolites are not straightforward. Complex tissue models involving multiple assumptions and approximations are required to translate MRI parameters into relevant biomedical information. For instance, assessment of absolute rates of tissue perfusion requires knowledge of the arterial input function [20,21]. Hence, the majority of physiological MRI applications use semiquantitative analysis (i.e., parameter values in the region of interest in relation to a reference tissue). An exemption remains cardiological applications, in which absolute values of functional parameters like stroke volume and ejection fraction can be derived by MRI, which is essentially based on morphometric measurements [22].

\section{IN VIVO MR TECHNIQUES IN DRUG RESEARCH}

In a simplified view, the drug discovery and development process can be divided in several steps (Fig. 1). In vivo MR techniques allows addressing relevant problems almost throughout the process.

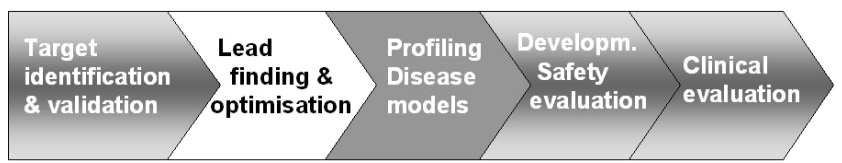

FIG. 1: Simplified view of the drug discovery process. In vivo MR techniques can play important roles in several steps, highlighted in grey. Transgenic mice may be used for target identification and validation, as disease models and for safety evaluations. Non-transgenic animals are used in disease models and for safety assessments. After a compound has been extensively determined to be safe in animal tests, its safety is assessed in healthy volunteers before large clinical efficacy studies can be started in patients.

\section{A. Target Identification and Validation}

Target selection, defined as the decision to focus drug discovery efforts on a specific (molecular) mechanism that is plausibly related to the disease process and, hence, anticipated to be of therapeutic value,,is influenced by considerations related to the underlying scientific rationale, medical need, and strategic/commercial aspects [23,24]. Target validation requires a causative link between a molecular/mechanistic drug target and a phenotype readout of the targeted disease. Genetically engineered animals are being increasingly used for this task [25,26]. Since mouse and man display striking similarities at the genetic level, it is likely that disease parameters observed in humans might be reproduced in mice. Correspondingly, phenotypic readouts in genetically altered mice might allow the prediction of relevant pharmacological effects in man. Evidently, characterization of transgenic animals in a non-invasive manner is an important task for MR techniques, which are particularly suited to analyze the structural and functional consequences of genotype expression. 


\section{B. Lead Finding, Validation and Optimization}

Once a target has been identified and validated, identification of a lead compound is the starting point for the development of new drug candidates. Today large compound libraries of typically $10^{6}$ compounds are screened using biochemical and cellular assays that have been made compatible for highly automated HTS. Primary hit compounds displaying activity exceeding a set threshold are further evaluated in secondary screening assays. The most promising hits are then selected for an often lengthy lead optimization program involving the synthesis and testing of compound analogues, during which issues such as target affinity, physico-chemical properties and aspects of compound safety are being addressed. In vivo MR techniques on the other hand do not have any function in this phase.

\section{Profiling Compounds in Animal Models of Diseases}

Lead optimization results in drug candidate compounds that need to be thoroughly evaluated in animal models of the human disease. The basic aim here is to obtain relevant information concerning drug efficacy, absorption, distribution, efficacy, metabolism and elimination. Both wildtype and transgenic animals, mostly small rodents, are used.

In view of a potential translation of methods to clinical drug testing non-invasive readouts of drug efficacy are preferable at this stage, yet not mandatory. In fact, the majority of pharmacological studies use invasive procedures. Nevertheless, there is an ethical motivation to use noninvasive methods such as imaging techniques, which contribute to the Three Rs (Reduce, Refine, Replace) principles used by Animal Ethics Committees in the governance of animal experimentation [27]. The use of imaging might be attractive from both an animal welfare and an economical point of view, since the number of animals required for a study can be reduced by up to $80 \%[15,18]$, a feature that is especially interesting in chronic studies, and in experiments involving transgenic species. Longitudinal study designs furthermore allow the reduction of inter-individual variances by using each animal as its own control, thereby enhancing the statistical power of experiments.

In the past, in vivo MR techniques have most extensively been used during compound profiling $[15,17,18]$. The general flow of activities is summarized in Fig. 2. For many diseases/disease models, a potential endpoint for the evaluation of the disease status or therapy efficacy is not readily accessible requiring the identification of biomarker, which is indicative of the disease status. Such biomarkers should have a clear disease relevance and should have predictive quality both with regard to spontaneous disease progression and potential therapy response. In addition, in order to facilitate translational activities, biomarkers used for preclinical studies should also be relevant for the human disease and clinical drug efficacy. Validation/qualification of the MR biomarker involves extensive comparison with established, usually invasive, readouts, in particular histology. In addition, they should correctly re- produce the well described effects of reference compounds. Only then may they be applied for non-invasive testing of novel drug candidate compounds.

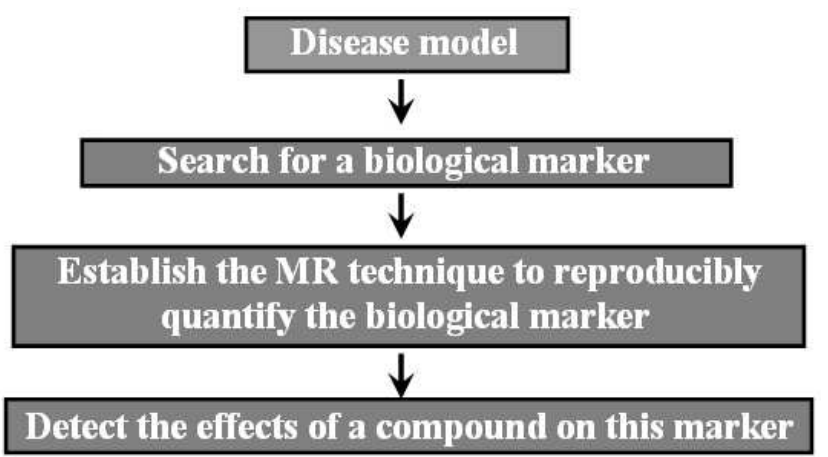

FIG. 2: General flow of activities for testing therapy efficacy in an animal model of disease.

\section{Safety Evaluation}

The same MR techniques used for evaluation of treatment efficacy in preclinical models of human diseases can also be applied to detect potential safety issues. The advantages of using MRI (and other non-invasive technologies) for toxicological studies are that effects can be studied in vivo (or post mortem) without the need for tissue dissection, sectioning and staining. Also, the occurrence and progression of potentially harmful structural and functional tissue alterations can be monitored in a longitudinal manner. Despite this attractive profile, the use of MRI, and imaging technologies in general, in drug safety studies has received little attention up to now. A main reason is the fact that toxicological studies used for regulatory authorities must be carried out following the guidelines for Good Laboratory Practice (GLP). Incorporation of MR techniques into routine toxicology programs running under GLP conditions will most likely require separate installations, since GLP compliance is usually not guaranteed in standard biological imaging laboratories. On the other hand, application of imaging techniques in experimental toxicology does not fall under these restrictions: studies in separate groups of animals aimed at establishing novel toxicological readouts or for internal decision making can be carried out in a straightforward manner. Such studies should be of great value to pharmacological research and will ultimately show whether imaging techniques might be used in a broader sense for evaluation of drug safety.

\section{E. Clinical Studies}

The aim of a novel therapy is to improve the patient's clinical status (endpoint). Clinical drug studies, in particular those addressing chronic disease, are time consuming and expensive. Similarly to the preclinical phase, biomarkers are a means to obtain early information on drug effectiveness and 
safety in clinical studies, which can lead to a substantial reduction in costs and development time [28,29]. More important is patient management: the time lost for a patient, who is not responsive to treatment, can be significantly reduced. A biomarker that substitutes a clinical endpoint is called a surrogate endpoint [30-33]. The most important criteria for valid surrogates are biological plausibility, a documented statistical relationship between the surrogate and an accepted clinical endpoint in epidemiological studies, and demonstration that treatment effects on the surrogate correspond to the clinical outcome.

In early clinical trials biomarkers can be used to demonstrate proof-of-concept of the pharmacological principle and to identify appropriate dose regimens for efficacy studies. both preclinical and clinical investigations are necessary to show a link between disease, pharmacological mechanism, and clinical endpoints.

The acceptance of biomarkers in general, and imaging biomarkers in particular by regulatory agencies is increasing [28], especially for oncology $[34,35]$. For example, for the 71 oncology drug approvals by the FDA in the period 1990-2002, end points other than survival were basis for approval in $68 \%$ (39 of 57) of applications granted regular approval and for all 14 applications granted accelerated approval [35]. The most common surrogate endpoint used was tumor response as determined by changes in tumor volume assessed by MRI or computerized tomography (CT).

The use of non-invasive readouts to assess therapy efficacy facilitates the translation from preclinical to clinical drug development In this regard, the non-invasive character of MR is a major asset. The potential of using the same readout in the preclinical and clinical phases of drug testing enhances the value of MR in pharmaceutical research. With the recent advances in the field of medical imaging, it is not surprising that the use of imaging biomarkers for the assessment of drug therapies is becoming more common. An imaging readout able to diagnose and characterize a disease state better than conventional methods will sooner or later be incorporated into clinical drug trials.

\section{MEASURING AT DIFFERENT SPATIAL SCALES}

The criteria imaging biomarkers must meet in order to be accepted by regulatory agencies are multifold. The validation/qualification process may involve not only clinical activities, but also extensive animal experimentation. Hence, transferability of images and protocols from small animal to human scanners and vice-versa becomes an issue. Sharing images from different platforms and manufacturers is necessary (see also section 6). The use of similar acquisition consoles in small animal and whole body systems is an option being evaluated by many manufacturers. Although this is an attractive approach for translational research, since it would considerably simplify the comparison of data obtained in different systems, it remains to be shown that protocol optimizations for human imaging are also applicable to e.g. mouse imaging. Some characteristics specifically related to the anatomy and physiology of every species could render this transition more challenging than a mere change of dimensional parameters in the acquisition protocols.

Spatial resolution constitutes a challenge when performing studies in small rodents. Voxel volumes have to be scaled with anatomical scales in order to accurately represent structures. Typical voxel volumes for a mouse brain study are about $3 \mathrm{nl}$ $\left(100 \times 100 \times 300 \mu \mathrm{m}^{3}\right)$, while a corresponding voxel in studies of human brain is $2 \mu \mathrm{l}\left(1000 \times 1000 \times 2000 \mu \mathrm{m}^{3}\right)$. The reduction in voxel volume by almost three orders of magnitude leads to a sensitivity issue: signal-to-noise ration in the mouse image would be reduced by a factor of 25 (square root of the ratio of voxel volumes) provided all other experimental parameters including the detector coil would be identical. Additionally, artifacts may be more severe when studying small structures. For example, effects of magnetic field inhomogeneities at tissue interfaces (bone/soft tissue), which are governed by the magnetic field strength and the change in magnetic susceptibility, but not by the anatomical dimension of the study object, will be more serious in mouse than in human images. Also, small rodents have higher respiratory and cardiac rates, which pose additional challenges when studying the thorax or abdomen.

Acquiring high quality images requires in many cases long examination times and interference with the animals' physiology. This is often incompatible with the reality of in vivo pharmacological studies in animals, in which the disease model and/or a compound may profoundly influence the physiology. The primary purpose of MRI in preclinical research is not to generate images of ultimate quality, but to allow the acquisition of data from which useful biomedical information can be derived with good reproducibility (and with a reasonable throughput). Obviously, MR techniques must be adapted to the biological situation, rather than to enforce the physiology for the sake of facilitating image acquisition. Since an MRI session always represents a certain burden for an animal, starting from the anesthesia, a careful balance between image quality and biological constraints should be envisaged [36]. As a general rule, the duration of an imaging session including animal preparation should be shorter than one hour.

\section{MOLECULAR IMAGING}

Questions commonly asked by drug developers include: where do drugs act in the body?; do they reach their target?; at what dose do side or even toxicological effects occur?; what organs are affected?; what are the optimal routes for drug delivery?; what is the receptor occupancy at a given dose level?; for how long does a compound stay bound? Addressing such questions demands for methods that allow visualization and quantification of molecular interactions at in the intact organism, so-called molecular imaging approaches [37-39]. Although the field is still in its infancy, novel imaging modalities and molecular probes are being developed at a rapid pace. It is therefore to be anticipated that drug developers will profit from such developments in a near future. 
Table 1 - In vivo imaging techniques currently used in the context of biomedical research and/or medical diagnosis.

\begin{tabular}{|c|c|c|c|c|c|c|}
\hline Technique & \begin{tabular}{|l|} 
Clinical \\
imaging
\end{tabular} & Resolution & $\begin{array}{l}\text { Animal } \\
\text { imaging }\end{array}$ & \begin{tabular}{|l|}
$\begin{array}{l}\text { Resolution and } \\
\text { time scale }\end{array}$ \\
\end{tabular} & Application & Main characteristics \\
\hline $\begin{array}{l}\text { SPECT (low energy } \\
\text { rays) }\end{array}$ & yes & 6-8 mm; s & yes & $1-2 \mathrm{~mm} ; \min$ & Functional & $\begin{array}{l}\text { radioisotopes have } \\
\text { longer half-lives than } \\
\text { those used in PET; } \\
\text { sensitivity } 10 \text { to } 100 \\
\text { times smaller than PET }\end{array}$ \\
\hline PET (high energy $\gamma$-rays) & yes & $4 \mathrm{~mm} ; \mathrm{s}$ & yes & $1-2 \mathrm{~mm} ; \min$ & $\begin{array}{l}\text { Metabolic, func- } \\
\text { tional, molecular }\end{array}$ & $\begin{array}{l}\text { High sensitivity (pico- } \\
\text { molar concentrations); } \\
\text { cyclotron needed }\end{array}$ \\
\hline CT & yes & $0.5 \mathrm{~mm} ; \mathrm{s}$ & yes & $50-100 \mu \mathrm{m} ; \min$ & $\begin{array}{l}\text { Anatomical, } \\
\text { functional }\end{array}$ & Poor soft tissue contrast \\
\hline Ultrasound & yes & $300-500 \mu \mathrm{m} ; \mathrm{s}$ & yes & $50 \mu \mathrm{m} ; \min$ & $\begin{array}{l}\text { Anatomical, } \\
\text { functional }\end{array}$ & $\begin{array}{l}\text { Difficulties to image } \\
\text { through bone or lungs; } \\
\text { microbubbles used for } \\
\text { contrast enhancement }\end{array}$ \\
\hline MRI & yes & $1 \mathrm{~mm}$; $\mathrm{s}$ to $\mathrm{min}$ & yes & $80-100 \mu \mathrm{m} ; \mathrm{s}$ to $\mathrm{h}$ & $\begin{array}{l}\text { Anatomical, func- } \\
\text { tional, molecular }\end{array}$ & $\begin{array}{l}\text { High spatial resolution } \\
\text { and soft tissue contrast }\end{array}$ \\
\hline Bioluminescence & no & - & yes & $\begin{array}{l}1-10 \mathrm{~mm} ; \mathrm{s} \text { to } \\
\mathrm{min}\end{array}$ & Molecular & $\begin{array}{l}\text { High sensitivity; } \\
\text { transgene-based ap- } \\
\text { proach; light emission } \\
\text { prone to attenuation } \\
\text { with increased tissue } \\
\text { depth }\end{array}$ \\
\hline Optical imaging & no & - & yes & $1-3 \mathrm{~mm}$; $\mathrm{s}$ to $\mathrm{min}$ & Molecular & $\begin{array}{l}\text { Excitation and emission } \\
\text { light prone to attenua- } \\
\text { tion with increased tis- } \\
\text { sue depth }\end{array}$ \\
\hline
\end{tabular}

\section{Abbreviations:}

ADMET absorption, distribution, metabolism, excretion and toxicology; CT computerized tomography; FDA Food and Drug Administration; GLP Good Laboratory Practice; HTS high-throughput screening; MR magnetic resonance; MRI magnetic resonance imaging; MRS magnetic resonance spectroscopy; PET positron emission tomography; SPECT single photon emission computed tomography

The most important imaging techniques potentially suited for providing molecular information in small animals are summarized in Table 1. In many respects the techniques are complementary; there is no 'all-in-one' imaging modality providing optimal sensitivity, specificity and temporo-spatial resolution. Due to its limited sensitivity, MRI is of limited value for detecting molecular processes in vivo; nevertheless, its high spatial resolution provides the exquisite anatomical reference for molecular data obtained with high sensitivity, low resolution imaging modalities. This might be achieved by postprocessing of data obtained in different imaging sessions or by simultaneous multimodality small animal imaging such as PET-MRI [40,41] and PET-CT [42]. Combining imaging data requires compatibility of data formats for the various modalities as well as sophisticated software tools for image coregistration (fusion), data visualization and integration across modalities.

Besides the imaging technique per se, a critical aspect of molecular imaging applications concerns the synthesis of appropriate target-specific probes (see [43] for a recent review on target-specific smart MR contrast agents). Most probes synthetized for molecular imaging will be limited to experimental research, since the approval process for human use involves similar hurdles as those for registering drugs [44]. Nevertheless, for target validation and assessments of drug distribution in animals, molecular imaging is going to play a relevant role in drug discovery. In order to take advantage of multimodality small animal imaging, the development of multimodal probes (e.g. for optical and MR imaging) is desirable [45].

\section{CONCLUDING REMARKS}

The impact that MR techniques may exert on the complex drug discovery process is basically determined by three factors:

(i) the multiparameteric contrast is of high diagnostic value increasing the chances to detect pathological transformation of tissue. Early disease detection enhances the probability of a successful therapeutic intervention;

(ii) accurate in vivo morphometric measurements allow sensitive and reproducible assessment of drug effects;

(iii) non-invasiveness allows the design of longitudinal study designs thereby increasing the statistical power.

There are significant differences with regard to opportunities and challenges for using MRI/S for the various disease areas/organs. Due to insufficient sensitivity, visualization of the drug per se is not feasible, i.e. questions concerning drug biodistribution, pharmacokinetic profiles, and drug-target in- 
teraction can, in general, not be addressed. Hence, MRI/S provide pharmacodynamic rather than pharmacokinetic information. However, coupled pharmacokinetic/pharmacodynamic studies could be carried out by combining MR with more sensitive molecular imaging approaches, such as PET [40] or optical imaging [46]. A crucial step for imaging to be fully integrated in drug research concerns the systematic and rigorous qualification of biomarkers via extensive correlation with well characterized and accepted reference data. Finally, standardization of imaging acquisition protocols and processing procedures, which will facilitate the comparison of data acquired at different sites, has to be propagated.

This huge task is going to keep researchers and clinicians busy for quite some time. The impact of their work will be the establishment of even more powerful diagnostic and prognostic tools that should translate into shortened drug development times. Not only the pharmaceutical industry is going to profit from such an effort, but also the larger medical community and, ultimately, the patients.

\section{Acknowledgement}

This contribution is dedicated with profound gratitude to Prof. Horacio Panepucci, with whom I had the great pleasure to work during my MSc studies in São Carlos. Horacio has instilled in me a passion for science, which is the driving force behind my daily activities, and has taught me the value of intuition in research. Being in his group, I had the privilege to witness, in March 1984, the formation of the first MR image acquired in the southern hemisphere, a feature that was realized primarily due to efforts by Alberto Tannús. I remember with great affection the long discussions in Horacio's office and lab, about the physics of imaging. Horacio's view that the pathway of physics is intimately linked to that of biology profoundly influenced my decisions and gave me sufficient impetus to pursue my own ways elsewhere. I owe Horacio a great deal of thankfulness.
[1] D. B. Kassel. Curr. Opin. Chem. Biol. 8, 339 (2004).

[2] NIH-FDA Conference: Biomarkers and Surrogate Endpoints: Advancing Clinical Research and Applications. Abstracts. Disease Markers 14, 187 (1998).

[3] G. Duyk. Science, 302, 603 (2003)

[4] B. M. Seddon and P. Workman. Br. J. Radiol. 76 (Spec 2), S128 (2003).

[5] A. Stahl, H. Wieder, M. Piert, H.J. Wester, R. SenekowitschSchmidtke, and M. Schwaiger. Mol. Imaging Biol. 6, 214 (2004).

[6] H. Horig and W. Pullman. J. Transl. Med. 2, 44 (2004).

[7] M. Rudin, N. Beckmann, and M. Rausch. Methods Enzymol. 385, 240 (2004).

[8] D. W. Klomp, H. V. Laarhowen, A. P. Kentgens, and A. Heerschap. Magn. Reson. Med. 50, 303 (2003).

[9] H. W. van Laarhoven, D. W. Klomp, Y. J. Kamm, C. J. Punt, and A. Heerschap. Cancer Res. 63, 7609 (2003).

[10] D. A. Hamstra, K. C. Lee, J. M. Tyhewicz, V. D. Schepkin, B. A. Moffat, M. Chen, K. J. Dornfeld, T. S. Lawrence, T. L. Chenevert, B. D. Ross, J. T. Gelovani, and A. Rehemtulla. Mol. Ther. 10, 916 (2004).

[11] Y. J. Kamm, A. Heerschap, E. J. van den Bergh, and D. J. Wagener. Anticancer Drugs 15, 229 (2004).

[12] J. R. Griffiths and J. D. Glickson. Adv. Drug Deliv. Rev. 41, 75 (2000).

[13] D. Artemov, M. Solaiyappan, and Z.M. Bhujwalla. Cancer Res. 61, 3039 (2001).

[14] M. Rudin, N. Beckmann, and A. Sauter. NMR Biomed. 12, 404 (1999).

[15] M. Rudin, N. Beckmann, R. Porszasz, T. Reese, D. Bochelen, and A. Sauter. NMR Biomed. 12, 69 (1999).

[16] M. Rudin, P. Allegrini, N. Beckmann, H.U. Gremlich, R. Kneuer, D. Laurent, M. Rausch, and M. Stoeckli. Ernst Schering Res. Found. Workshop 48, 47 (2004).

[17] N. Beckmann, T. Mueggler, P.R. Allegrini, D. Laurent, and M. Rudin. Anat. Rec. 265, 85 (2001).

[18] N. Beckmann, B. Tigani, D. Laurent, R. Panizzutti, and M. Rudin. Drug Discov. Today 9, 35 (2004).

[19] N. Beckmann, B. Tigani, L. Mazzoni, and J. R. Fozard. Trends
Pharmacol. Sci. 24, 550 (2003).

[20] R. M. Weisskoff, D. Chesler, J. L. Boxeman, and B. R. Rosen. Magn. Reson. Med. 29, 553 (1993).

[21] M. Rudin, N. Beckmann, and A. Sauter. Magn. Reson. Imaging 15, 551 (1997)

[22] M. Rudin, P. Birgit, K. Umemura, and W. Zierhut. Basic Res. Cardiol. 86, 165 (1991).

[23] R. L. Stein. Drug Discov. Today 8, 245 (2003).

[24] J. Knowles and G. Gromo. Nat. Rev. Drug Discov. 2, 63 (2004).

[25] J. Tornell and M. Snaith. Drug Discov. Today 7, 461 (2002).

[26] B. P. Zambrowicz and A. T. Sands. Nat. Rev. Drug Discov. 2, 38 (2003)

[27] C. A. Schuppli, D. Fraser, and M. McDonald. Altern. Lab. Anim. 32, 525 (2004).

[28] R. Frank and R. Hargreaves. Nat. Rev. Drug Discov. 2, 566 (2003).

[29] J. J. Smith, A. G. Sorensen, and J. H. Thrall. Radiology 227, 633 (2003)

[30] W. A. Colburn. J. Clin. Pharmacol. 40, 1419 (2000).

[31] L. J. Lesko, M. Rowland, C. C. Peck, and T. F. Blaschke. J. Clin. Pharmacol. 40, 803 (2000).

[32] L. J. Lesko and A. Atkinson, Ann. Rev. Pharmacol. Toxicol. 41, 347 (2001)

[33] H. H. Pien, A. J. Fischman, J. H. Thrall, and A. G. Sorensen, Drug Discov. Today 10, 259 (2005).

[34] G. J. Kelloff and C. C. Sigman, Eur. J. Cancer 41, 491 (2005).

[35] J. R. Johnson, G. Williams, and R. Pazdur. J. Clin. Oncol. 21, 1404 (2003).

[36] L. A. Colby and B. J. Morenko, Comp. Med. 54, 623 (2004).

[37] M. Rudin and R. Weissleder, Nat. Rev. Drug Discov. 2, 123 (2003).

[38] T. F. Massoud and S. S. Gambhir, Genes Dev. 17, 545 (2003).

[39] I. J. Hildebrandt and S. S. Gambhir, Clin. Immunol. 111, 210 (2004).

[40] R. B. Slates, K. Farahani, Y. Shao, P. K. Marsden, J. Taylor, P. E. Summers, S. Williams, J. Beech, and S. R. Cherry. Phys. Med. Biol. 44, 2015 (1999).

[41] H. Benveniste, J. S. Fowler, W. Rooney, Y. S. Ding, A. L. Baumann, D. H. Moller, C. Du, W. Backus, J. Logan, P. Carter, J. 
D. Coplan, A. Biegon, L. Rosenblum, B. Scharf, J. S. Gatley, and N. D. Volkow. J. Nucl. Med. 46, 312 (2005).

[42] F. H. Fahey. Neuroimaging Clin. N. Am. 13, 659 (2003).

[43] E. J. Delikatny and H. Poptani, Radiol. Clin. North Am. 43, 205 (2005).

[44] F. A. Jaffer and R. Weissleder, JAMA, 293, 855 (2005).
[45] M. Doubrovin, I. Serganova, P. Meyer-Kuckuck, V. Ponomarev, and R.G. Blasberg. Bioconjug. Chem. 15, 1376 (2004).

[46] V. Ntziachristos, J. Ripoll, L. V. Wang, and R. Weissleder. Nat. Biotechnol. 23, 313 (2005). 